\title{
Case Report \\ Acute Cholecystitis Caused by Ceftriaxone Stones in an Adult
}

\author{
Christian D. Becker ${ }^{1}$ and Robert A. Fischer ${ }^{2}$ \\ ${ }^{1}$ The Mount Sinai Medical Center, New York, NY 10029, USA \\ ${ }^{2}$ Albert Einstein Medical Center, Philadelphia, PA 19141, USA \\ Correspondence should be addressed to Christian D. Becker, christian.d.becker@gmail.com
}

Received 26 January 2009; Accepted 24 March 2009

Recommended by Gianfranco D. Alpini

Acute cholecystitis is a major health problem. There are multiple etiologies to be considered and early recognition of the condition is important to optimize management and outcome. We report the first case in the medical literature of symptomatic acute cholecystitis triggered by ceftriaxone-associated gallbladder sludge formation and, importantly, solid ceftriaxone gallstone formation in an adult patient with underlying mineral and pigment cholecystolithiasis, necessitating cholecystectomy. This case serves as a reminder for physicians to keep this uncommon cause of cholecystolithiasis and cholecystitis in mind in patients who receive prolonged ceftriaxone therapy. These patients should be cautioned to promptly report to their physicians any signs or symptoms of cholecystitis in order to ensure timely and appropriate evaluation.

Copyright ( 2009 C. D. Becker and R. A. Fischer. This is an open access article distributed under the Creative Commons Attribution License, which permits unrestricted use, distribution, and reproduction in any medium, provided the original work is properly cited.

\section{Background}

Acute cholecystitis is a major health problem. There are multiple etiologies to be considered and early recognition of the condition is important to optimize management and outcome.

\section{Case Report}

A-68-year-old African-American male was admitted for 2 days of worsening right upper quadrant abdominal pain. The pain was constant, noncolicky, nonradiating, 6-8/10 in intensity, accompanied by nausea and vomiting with attempted food intake. No fevers or chills were present.

9 weeks prior to admission the patient underwent craniotomy for drainage of multiple brain abscesses. Cultures were negative. Long-term empiric therapy with high doses of ceftriaxone (2 gm i.v. q12 hours), caspofungin (50 mg i.v. q24 hours), metronidazole (500 $\mathrm{mg}$ p.o. q8 hours), and corticosteroids was initiated at that time.

Physical examination revealed marked right upper quadrant tenderness and a positive Murphy's sign. Laboratory data showed a normal white blood cell count of $5400 / \mathrm{mm}^{3}$, hemoglobin of $10.3 \mathrm{~g} / \mathrm{dL}$, normal blood chemistry values except a blood glucose of $157 \mathrm{mg} / \mathrm{dL}$. The total bilirubin was normal at $0.2 \mathrm{mg} / \mathrm{dL}$, the alkaline phosphatase was slightly elevated at $177 \mathrm{IU} / \mathrm{L}$. The transaminases were normal (AST $21 \mathrm{IU} / \mathrm{L}, \mathrm{ALT} 14 \mathrm{IU} / \mathrm{L})$.

A CT scan revealed gallbladder wall thickening, pericholecystic fluid, and multiple gallstones in the gallbladder.

The patient underwent cholecystectomy on the day of admission. The gallbladder contained numerous greenyellow gallstones of various sizes (see Figure 1), which were soft and easily compressible.

The patient experienced an uncomplicated postoperative course.

The prolonged treatment with high doses of ceftriaxone and peculiar consistency of the gallstones resulted in the clinical suspicion of ceftriaxone-associated gallstones. Therefore, a gallstone sample was analyzed by high-pressure liquid chromatography [1]. The sample contained $70.4 \%$ minerals, $7.5 \%$ pigments, and $0.1 \%$ cholesterol as well as significant amounts of ceftriaxone calcium salt (mean of $22 \%$, with 3 separate measurements of $20.97 \%, 23.43 \%$, and $21.59 \%$ ).

\section{Discussion}

Ceftriaxone is $10-40 \%$ hepatically eliminated [2] and is known to induce reversible precipitates of ceftriaxone calcium in the gallbladder [3]. Ultrasonographically, these 


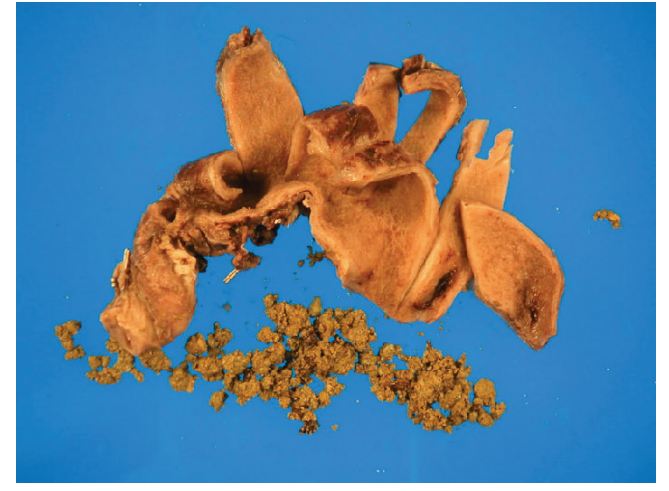

Figure 1: Surgical specimen (gallbladder, with resection clips) showing a large number of greenish-brownish gallstones of various sizes and "crumbly" consistency.

precipitates resemble gallbladder sludge, but the ultrasoundmorphologic similarity to gallstones as well as the rapid disappearance after discontinuation of ceftriaxone has resulted in the term "ceftriaxone pseudolithiasis."

The phenomena of ceftriaxone-associated sludge formation and ceftriaxone-associated cholelithiasis are well documented in the pediatric literature and have resulted in unnecessary cholecystectomies in pediatric patients. The incidence of ceftriaxone sludge formation in patients treated with ceftriaxone ranges from $25 \%$ to $46 \%$, but only a minority of these patients become symptomatic [4].

A Medline search with the terms ("ceftriaxone" AND ("acute cholecystitis" OR "cholecystolithiasis")) from 1980 to 2008 revealed one report of ceftriaxone-associated cholecystolithiasis leading to acute gallstone pancreatitis in a 42year-old male [5], but no reports of ceftriaxone-associated cholecystolithiasis with acute cholecystitis in adults.

However, there are several reports of coexisting cholecystolithiasis in patients with ceftriaxone-associated gallbladder sludge formation. There are three mechanisms by which a patient receiving ceftriaxone can develop acute cholecystitis: ceftriaxone-associated sludge can trigger existing gallstones to become symptomatic, ceftriaxone pseudolithiasis can transform into ceftriaxone gallstones, or the patient can become symptomatic from preexisting cholecystolithiasis unrelated to ceftriaxone therapy.

Because of the unusual appearance and consistency of the stones in our patient and the analysis showing that ceftriaxone was incorporated into the stones in significant amounts, we believe that ceftriaxone therapy was responsible for the development of acute cholecystitis in our patient.

\section{Conclusion}

We report the first case in the medical literature of symptomatic acute cholecystitis triggered by ceftriaxoneassociated gallbladder sludge formation and, importantly, solid ceftriaxone gallstone formation in an adult patient with underlying mineral and pigment cholecystolithiasis, necessitating cholecystectomy.
This case serves as a reminder for physicians to keep this uncommon cause of cholecystolithiasis and cholecystitis in mind in patients who receive prolonged ceftriaxone therapy. These patients should be cautioned to promptly report to their physicians any signs or symptoms of cholecystitis in order to ensure timely and appropriate evaluation.

\section{Acknowledgment}

The authors thank Dr. Haigh and Dr. Savard, University of Washington, Seattle, USA for the high-pressure liquidchromatography analysis of the gallstone sample.

\section{References}

[1] C. Y. Chan, K. Chan, and G. L. French, "Rapid high performance liquid chromatographic assay of cephalosporins in biological fluids," The Journal of Antimicrobial Chemotherapy, vol. 18 , no. 4, pp. 537-545, 1986.

[2] P. J. McNamara, K. Stoeckel, and W. H. Ziegler, "Pharmacokinetics of ceftriaxone following intravenous administration of a $3 \mathrm{~g}$ dose," European Journal of Clinical Pharmacology, vol. 22, no. 1, pp. 71-75, 1982.

[3] U. B. Schaad, H. Tschäppeler, and M. J. Lentze, "Transient formation of precipitations in the gallbladder associated with ceftriaxone therapy," Pediatric Infectious Disease, vol. 5, no. 6, pp. 708-710, 1986.

[4] M. L. Shiffman, F. B. Keith, and E. W. Moore, "Pathogenesis of ceftriaxone-associated biliary sludge: in vitro studies of calcium-ceftriaxone binding and solubility," Gastroenterology, vol. 99, no. 6, pp. 1772-1778, 1990.

[5] A. J. Lopez, P. O’Keefe, M. Morrissey, and J. Pickleman, "Ceftriaxone-induced cholelithiasis," Annals of Internal Medicine, vol. 115, no. 9, pp. 712-714, 1991. 


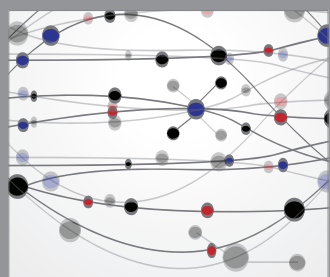

The Scientific World Journal
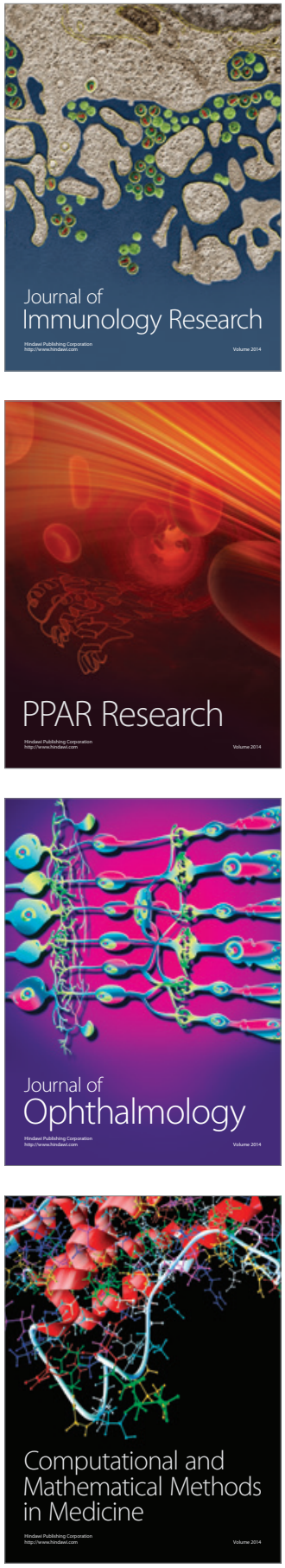

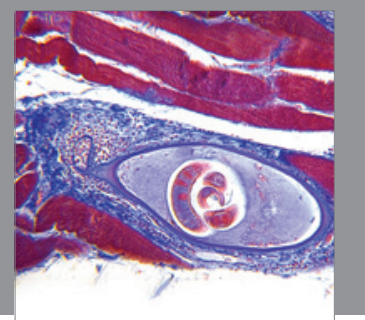

Gastroenterology

Research and Practice
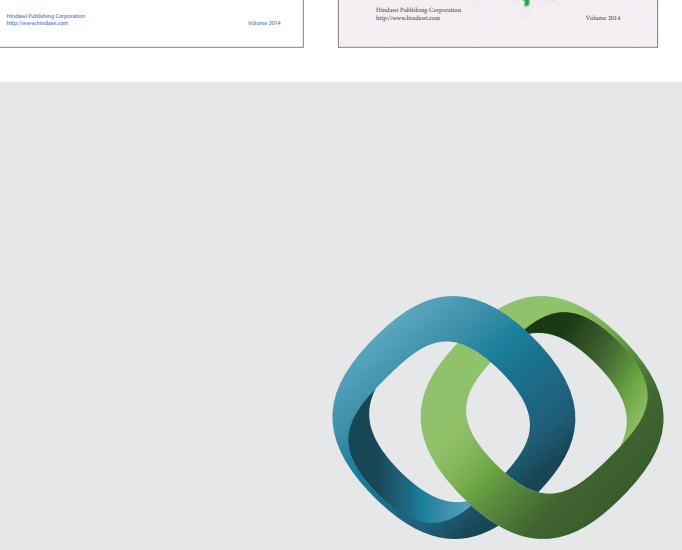

\section{Hindawi}

Submit your manuscripts at

http://www.hindawi.com
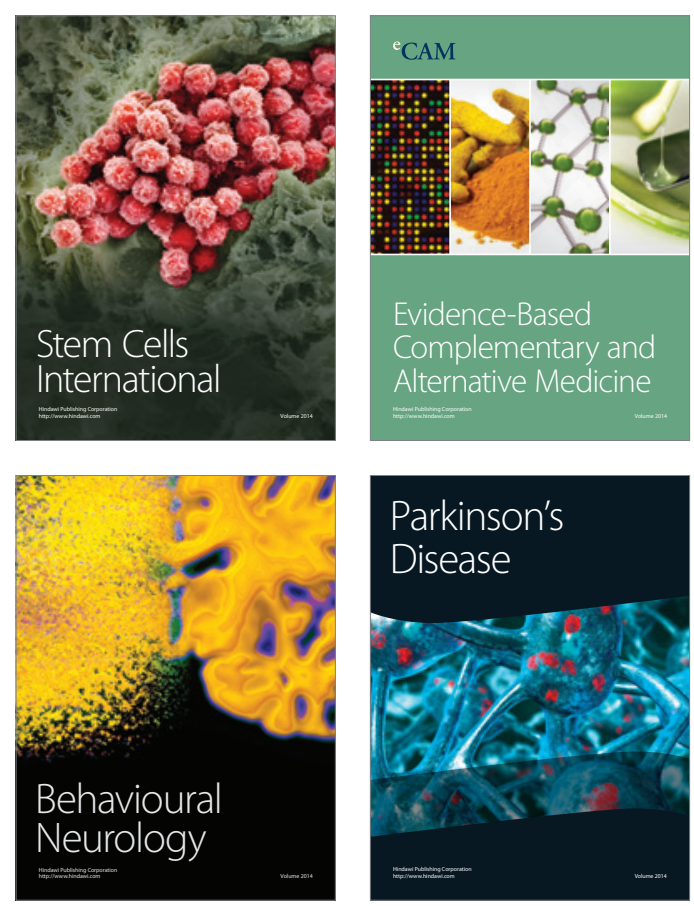

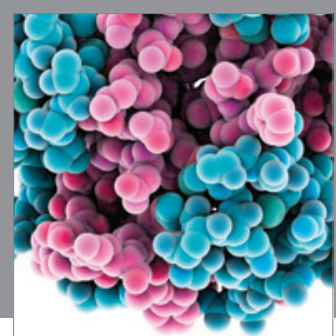

Journal of
Diabetes Research

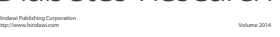

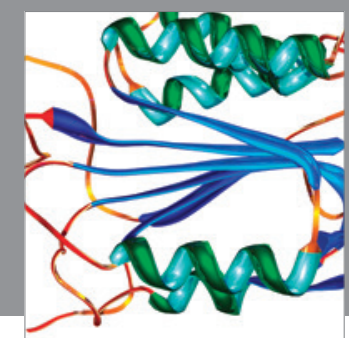

Disease Markers
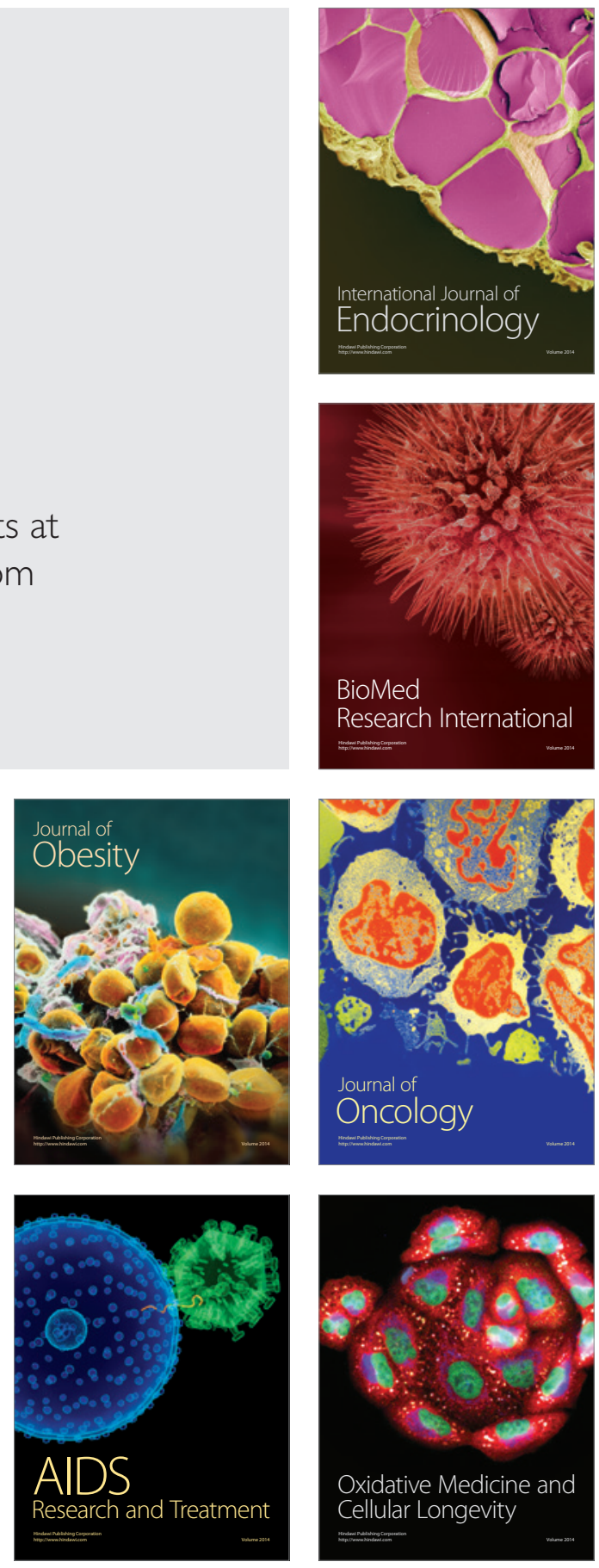